corneal ulcer was detected. These events prompted a xerophthalmia survey in the six feeding centres of the camps. In each feeding centre all children present on the day of the survey were examined clinically by the author. Risk factors were also investigated. One thousand five hundred and six children were examined. Bitot's spot was seen in four children, conjunctival xerosis in seven, corneal xerosis in one, and corneal ulcer in two. The children attending the two feeding centres serving the population of newcomers had a significantly higher risk of xerophthalmia (prevalence ratio $4.8, p>0.01$ ), as did the children with history of diarrhoea in the previous month (prevalence ratio $1 \cdot 7$, $\mathrm{p}>0.05)$.

The $0.3 \%$ prevalence of Bitot's spot and $0.2 \%$ temporary corneal lesions indicate that this condition is an important health problem in the refugee population investigated, not only because of the risk of blindness but also because vitamin A deficiency is associated with increased mortality mainly from diarrhoea, ${ }^{12}$ the major cause of death in the camps. The absence of cases of corneal scar suggests that xerophthalmia is a recent event in this population associated with refugee life, otherwise this permanent lesion would be about five times more frequent than the temporary corneal lesions, because of accumulation. ${ }^{1}$ Therefore, the prevalence of this condition should increase over time unless corrective measures are taken. Vitamin A distribution associated with vaccination at registration was strengthened. The history of risk factors for this condition was introduced in the feeding centres for newly admitted children and the at risk population examined for signs of vitamin A deficiency. The Ngara refugee camps are relatively well organised, but xerophthalmia may well affect other refugees and displaced Rwandan people in other camps.

G DE SOLE 1 Pascoe Avenue, BP 6988, Harare, Zimbabwe

1 WHO, UNICEF, USAID, HKI IVACG. Control of vitamin A deficiency and xerophthalmia. 1982 Technical Report Series 672. Geneva: 1982.

2 Sommer A, Hussaini G, Tarwotjo I, Susanto D. Increased mortality in children with mild vitamin A deficiency. Lancet 1983; ii: 585-8.

\section{Serious eye injury caused by rotating wire brushes}

EDITOR,-Hassett ${ }^{1}$ reported a series of cases highlighting the risk of ocular injury from rotating wire brushes. We wish to emphasise this risk and also remind colleagues that such injuries may lead to serious intraocular infection.

A 37-year-old man sustained a corneoscleral perforation by a piece of wire from a rotating brush. He immediately removed the $3 \mathrm{~cm}$ long fragment before presenting to the casualty department. At presentation, visual acuity (VA) was $6 / 12$ in the affected eye. Funduscopy and $x$ ray investigations revealed no evidence of retained intraocular foreign body (IOFB). The anterior chamber (AC) was formed, and slit-lamp examination showed a microhyphaema. The wound was not self sealing and primary repair was performed within 24 hours of the injury.

On the first postoperative day a hypop on developed, accompanied by a fibrinous pupillary membrane and intense AC activity. Vitreous biopsy and AC tap provided samples from which a heavy growth of coagulase negative Staphylococcus aureus was shown. An intravitreal injection of vancomycin and gentamicin was given, and intensive topical administration of these antibiotics commenced. The signs of infection settled, but a mature cataract developed after 2 weeks.

Six weeks after the injury, he underwent further surgery, combining complete vitrectomy with cataract extraction and capsular fixated posterior chamber lens implantation. After 8 weeks the visual acuity had returned to $6 / 36$.

The overall rate of endophthalmitis secondary to infection following penetrating injury is less than $10 \% .^{2}$ Penetrating eye injuries caused by high velocity projectiles such as hardened steel fragments from cold chisels are thought to carry a low risk of infection. This is because the particles attain very high temperatures before penetration and therefore sterilise themselves.

Wire brushes are not necessarily at a high temperature when they disintegrate and, as demonstrated in our case, may introduce infection. Therefore we recommend that injuries resulting from the use of rotating wire brushes should be treated with caution, as there is a definite risk of subsequent endophthalmitis.

\section{N R HAWISWWAN Department of Ophthalmology, clo Ward A4, University Hospital of Wales, Heath Park, Cardiff CF $44 X W$}

1 Hassett P, Cleary PE. Serious eye injuries caused by rotating wire brushes. $\mathrm{Br} \mathcal{F}$ Ophthalmol 1994 78: 491 .

2 Thompson JT, Parver LM, Enger CL, Miele WF, Liggett PE. Infectious endophthalmitis after penetrating injuries with retained intraocular foreign bodies. National Trauma Eye System. Ophthalmology 1993; 100: 1468-74.

\section{Reply}

EDITOR,-We have read, with interest, of the case described by Chan and Hawksworth. It confirms the serious nature of these injuries. Although intraocular infection did not occur in the cases that we described, clearly there is a risk of endophthalmitis. This further emphasises the importance of the safety precautions that we have recommended.

P E CLEARY P HASSET Department of Ophthalmology Cork Regional Hospital, Wilton, Cork, Ireland

\section{Automated ESR analysis in 20 minutes}

EDITOR,-Significant benefits to patients and staff can be obtained by performing erythrocyte sedimentation rate (ESR) analysis in 20 minutes in the eye department. Faster sedimentation is possible because of the Boycott phenomenon, whereby erythrocytes sediment more quickly when streaming down the wall of an angled tube than when falling vertically. This property is utilised in the Ves-Matic system (Biomen diagnostics, Croydon, Surrey CR0 2BN). Premanufactured tubes containing a standard diluent concentration are vacuum filled directly from the venepuncture site and placed into individual holders in the measuring device which maintains the tubes at an angle of $18^{\circ}$ to the vertical. A photoelectric cell records the height of the column of erythrocytes before and after sedimentation. The time remaining for the analysis to be complete is displayed, followed by the result, until the next measurement is initiated.

We compared the performance of this portable machine with our laboratory's 'Seditainer' (Beckton Dickinson Vacutainer systems, Cowley, Oxford), ESR method. Seventy patients who required ESR estimation had two blood samples taken from a single venepuncture, for analysis by the above methods. The two measurements showed excellent correlation $(x=8 \cdot 6+1 \cdot 15 y$, $\mathrm{p}=0.93$ ).

Three Ves-Matic measuring devices are available: Mini, Junior, and Senior, holding 4, 20 , and 60 samples respectively, plus facility for hard copy printout and connection to the laboratory computer. The apparatus is portable, gives a quick accurate result, is simple and convenient to use, and uses a closed system for venepuncture and analysis. It offers a significant time saving to both staff and patients.

$$
\begin{array}{r}
\text { P A HARVEY } \\
\text { R TANDON } \\
\text { A K SINGH } \\
\text { University Department of Ophthalmology and } \\
\text { Orthoptics, } \\
\text { Royal Hallamshire Hospital, } \\
\text { Sheffield S10 } 2 f F
\end{array}
$$

\section{Ophthalmoscopic sign of early keratoconus}

EDITOR,-I read with interest the recent letter by Pathmanathan et al, ${ }^{1}$ about the ophthalmoscopic sign of early keratoconus.

This sign is known to some ophthalmologists and optometrists who use it regularly in the assessment of suspected cases of keratoconus at Moorfields Eye Hospital, London, as 'the oildrop sign', because of the disruption of the red reflex by a circular, dark or reddishbrown central shadow which looks like an oildrop.

The sign is best seen through a dilated pupil with $a+5$ lens in the direct ophthalmoscope held at $33-50 \mathrm{~cm}$ from the observer to the patient's eye ${ }^{2}$ and is almost diagnostic of keratoconus.

Since, however, changes in the refractive index of the lens or early nuclear cataract can be confused with keratoconus when the sign is elicited, it would be desirable to evaluate the cornea from the temporal side also, to eliminate any reflex coming from the lens.

I N NARTEY Moorfields Eye Hospital, City Road, London EC1V 2PD

1 Pathmanathan T, Falcon MG, Reck A Ophthalmoscopic sign of early keratoconus. $B$ f Ophthalmol 1994; 78: 510 .

2 Gasset AR. Keratoconus. In: Gasset AR, ed. Contact lenses and corneal disease. New York: Appleton Century Crofts, 1976: 339. 